\author{
SERIES 'OCCUPATIONAL ASTHMA' \\ Edited by C. Mapp
}

\title{
Animal models of occupational asthma
}

\author{
M.H. Karol
}

Animal models of occupational asthma. M.H. Karol. CERS Journals Ltd 1994.

ABSTRACT: Occupational asthma is characterized by variable airflow obstruction occurring in the workplace. The presence of airways inflammation and hyperreactivity provides further evidence for the disease. Since its pathogenic mechanism(s) are unknown, animal models have been developed to investigate the various disease processes, as well as to enable study of environmental and genetic factors which may contribute to disease development.

Numerous parameters can be measured in animal systems, including specific and total immunoglobulin E (IgE), pulmonary eosinophilia, diaphragm contractions and airflow muscle hypertrophy. It is recognized that no single factor is sufficient to lead to a conclusion of occupational asthma, but rather that a selected combination of parameters is most fitting. Animal species selected for study have included: mice, rats, guinea-pigs, rabbits, sheep, horses and nonhuman primates. A guinea-pig system has been utilized for more than 90 yrs and has contributed to the basic understanding of physiological and immunological processes involved in allergic respiratory sensitization. The benefits as well as the disadvantages to be derived from each of the animal systems have been enumerated in this review.

Certain caveats must be recognized in using animal systems. Attention must always be given to identifying differences which exist between animal and human systems, including morphological, physiological and immunological factors. The extent of bronchus-associated lymphoid tissue (BALT) differs greatly among animal species and probably plays a major role in development of allergic responsiveness in animals. In using animal systems, it must be appreciated that animals are only surrogates. Results from such studies must be compared with information obtained from clinical evaluation in order to avoid faulty extrapolations. Prudent employment of animal models is expected to advance the recognition, treatment and prevention of occupationally-based asthma.

Eur Respir J., 1994; 7, 555-568.
Center for Environmental and Occupational Health and Toxicology, University of Pittsburgh, Pittsburgh, PA, USA.

Correspondence: M.H. Karol

Center for Environmental and Occupational Health and Toxicology

University of Pittsburgh

PA

USA

Keywords: Airway hyperreactivity cytokines

early-onset response

environment and genetic influences

late-phase response

pathogenic mechanisms

Received: July 201993

Accepted for publication December 231993

The article was supported under grant No. ES 05651 from the National Institute of Environmental Health Sciences.
Asthma is a chronic disease characterized by acute exacerbations of coughing, dyspnoea, wheezing and chest tightness. Patients usually have reduced forced expiratory volume in one second $\left(\mathrm{FEV}_{1}\right)$ as well as reduced airflow. Other features characteristic of asthma but not unique to the disease are airway inflammation and bronchial hyperresponsiveness. Evidence of reversible airways obstruction confirms the diagnosis. Occupational asthma is diagnosed by first establishing the diagnosis of asthma, and then establishing that the asthma is due to occupational exposure.

Heightened airways responsiveness to a wide variety of pharmacological and physical agents is characteristic of asthma. The degree of airway responsiveness usually correlates with the severity of the disease [1]. Because of this association, measurement of airway responsiveness should be given extra attention in development of animal models for the chronic disease, asthma.

The pathogenic mechanisms of occupational asthma remain unclear, as does the role of environmental and genetic factors in the disease process. Based on the relationship between airway hyperreactivity and serum immunoglobulin E ( $\operatorname{IgE})[2,3]$, it has been suggested that all asthma has an atopic component, where atopy is defined as the presence of elevated IgE. Where atopy is defined by skin tests, two-thirds of patients with asthma are atopic [4].

Asthma appears to be under the influence of both genetic and environmental factors. A plan depicting the possible role of these factors is presented in figure 1 [5]. Familial studies have provided evidence for a genetic component in development of allergy. Inheritance of atopy appears to be dominant and associated with a specific gene on the short arm of chromosome 11 [6]. Linkage is expressed predominantly through the mother; genes derived from the father appear not to be expressed.

Environmental exposure to the offending allergen is necessary for the expression of genes to the allergen. Importantly, the level of allergen exposure governs the expression of the gene(s) [5].

Sensitization appears to be favoured when allergen is presented in the presence of irritants, such as pollutants, or with upper respiratory viral infections [7]. The mechanism of this environmentally contributed adjuvant 


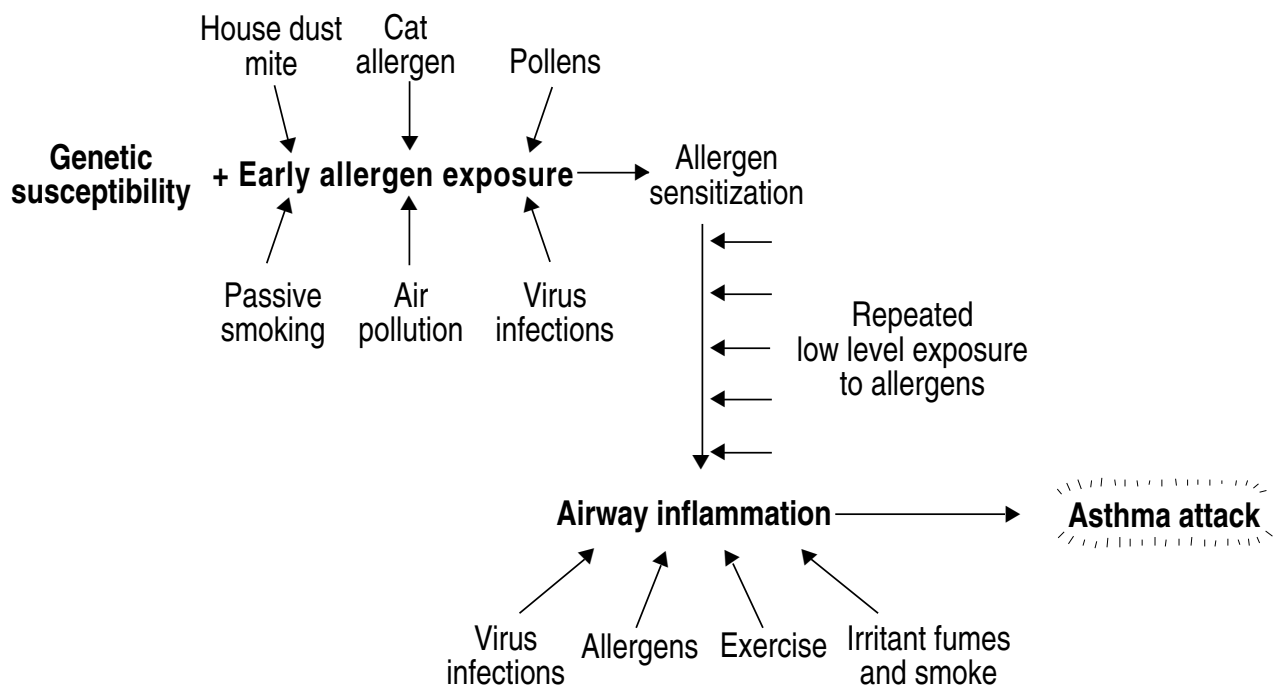

Fig. 1. - Plan depicting potential influence of genetic and environmental factors on development of asthma. From [5], with permission.

activity is unclear, but has been hypothesized to occur through inflammation and enhanced penetration of allergen through mucosal barriers [7].

\section{Characteristics of occupational asthma}

Although occupational asthma is currently the most prevalent occupational lung disease [8], its pathogenesis remains unclear. To develop animal models with which to gain increased understanding of the disease process, it is essential to start with a clear definition of the disease and its clinical manifestations. Occupational asthma is frequently defined as variable airflow obstruction caused by a specific agent in the workplace [8]. The definition has been modified by some to require a latent period between onset of workplace exposure to the substance and occurrence of the disease. Furthermore, the existence of bronchial hyperresponsiveness or eosinophilia has been added to the definition to distinguish it from other conditions, such as byssinosis, where there is only reversible airflow obstruction.

A listing of clinical findings in occupational asthma is given in table 1. Severity of asthma is based on the frequency and intensity of symptoms. The mildest grade is characterized by acute attacks upon allergen exposure, and with symptoms reversed by $\beta$-adrenergic agonists [9]. Lung function is normal between acute attacks. More severe grades of asthma are characterized by: a sustained increase in airway resistance (the late-phase response); impaired basal lung function; and heightened airways responsiveness to nonspecific irritants (hyperreactivity). Cellular inflammation is common and comprised of increased numbers of mast cells and eosinophils in the bronchial mucosa [10]. The inflammation is believed to contribute both to the late-phase response and to the heightened airway reactivity.

Regarding the mechanisms underlying occupational asthma, convincing evidence has been presented for humoral immunological aetiology of the early-onset response. This response is believed to result from mediators released
Table 1. - Clinical findings in occupational asthma

Coughing, wheezing, dyspnoea

Chest tightness

Airflow disturbance occurring either immediately following, or hours after, exposure to allergen

Impaired basal lung function (in severe cases)

Bronchial hyperreactivity to pharmacological agents

Eosinophilic airway inflammation

Increased mast cells in the bronchial mucosa

Activated T-cells in the bronchial mucosa

Elevation of serum $\operatorname{IgE}$, both antigen-specific and total

IgE: immunoglobulin E.

by activated granulated cells, such as mast cells [5]. The rapid response is reversed by $\beta_{2}$-agonists, and probably results from contraction of airway smooth muscle, which has receptors for the released mediators. The crosslinking of mast cell-bound IgE is followed by release of tumour necrosis factor- $\alpha$ (TNF- $\alpha$ ) and interleukin-4 (IL4). This suggests involvement of T-cells in an early stage of response.

The association of atopy and $\mathrm{IgE}$ with most cases of childhood and young adult asthma is accepted. On the other hand, the lack of association of either atopy or IgE with occupational asthma to low molecular weight (LMW) allergens suggests consideration be given to differences in basic mechanisms of occupational asthma to high versus low molecular weight agents.

The late-phase response is characterized by inflammation. When associated with occupational asthma, the inflammation consists of infiltration of airway walls with eosinophils, neutrophils, plasma cells and lymphocytes [10]. The airways appear free of fibrosis. Histological evaluation provides evidence of a subacute process rather than of a chronic disease. Small biopsy specimens from patients with allergic asthma have indicated mast cells and eosinophils as the predominant cell types. Mast cells are increased in the bronchial epithelium, and there is evidence of mast cell degranulation. Eosinophils are thought to be the major leucocyte effector cell and appear 
degranulated in electron micrographs [11]. The inflammatory response is believed to develop as a result of eosinophil-derived protein, together with reactive oxygen radicals, damaging the bronchial tissue.

T-cells appear to have a major role in the inflammation. Many of the elements of the late-phase response, including the eosinophilia, mast cell activation and IgE production appear to be regulated by T-lymphocytes. Atopic asthmatics have been found to possess activated T-cells in their airways [12]. T-cells in bronchoalveolar lavage (BAL) fluid have increased expression of surface CD25 (IL-2R) and human leucocyte antigen (HLA)-DR. In addition, they express messenger ribonucleic acid (mRNA) for cytokines known to play a part in recruitment and activation of mast cells and eosinophils (i.e. IL-3, IL-4, IL-5 and granulocyte/macrophage colony-stimulating factor (GM-CSF)). They probably regulate inflammation through their production of cytokines, including IL-4 and IL-5.

Additional evidence of T-cell regulation of the inflammatory response has been obtained from animal studies. Antigen-induced eosinophil recruitment into the tissue of sensitized mice was shown to be mediated by CD4+ T-cells and IL-5 [13]. Administration of interferon- $\gamma$ $($ IFN- $\gamma$ ) prevented the antigen-induced infiltration of eosinophils into the lung of sensitized mice and decreased the antigen-induced CD4+ T-cell infiltration into the trachea. These results suggest a role for IFN- $\gamma$ in modulation of the late-phase response, and specifically in reduction of eosinophilic inflammation.

Mast cells are also believed to have a central role in the inflammatory process. Mediators released from this cell type can cause bronchial smooth muscle contraction, and induce migration of neutrophils and eosinophils to the site of degranulation.

\section{Rationale for development of animal models}

Animal models are typically developed for either research and/or testing purposes. They are necessary to permit prediction of the sensitization capability of new chemicals before the latter reach large scale production. For occupational asthma, animal models are particularly needed because of our current incomplete understanding of the mechanisms of chemical sensitization.

Animal models have been able to reproduce both the acute airway spastic response associated with occupational asthma, as well as the late-onset inflammatory reactions. Many have also demonstrated hyperreactive airways (table 2). Animal models can be particularly effective in elucidating factors associated with development of airway sensitivity and expression of responses. Animal experimentation has enabled identification of the importance of antigen dose (or extent of antigen exposure) to the processes of sensitization and elicitation of responses [14, 15].

Animal models are ideally suited to investigation of the role of environmental factors and of genetic predisposition in development of asthma. Genetic transmission has been suggested for allergen skin test reactivity, levels of total serum $\mathrm{IgE}$, pulmonary function measurements and asthma [16].
Table 2. - Parameters appropriate for study of asthma in animal models

Airway hyperresponsiveness

Elevated IgE, or other anaphylactic antibody class

Pulmonary eosinophilia

Mast cell, and/or eosinophil derived products in the bronchial mucosa or bronchoalveolar lavage

Diaphragmatic contractions

Cyanosis

Lung resistance

Microvascular leakage of protein

Fever

Elevated ratio of interleukin-4: interferon- $\gamma$

Increased airway resistance

Decreased dynamic compliance

Abnormal airflow

Airway mucus

Airway smooth muscle hypertrophy

IgE: immunoglobulin E.

\section{Parameters which define occupational asthma}

In developing an animal model, it is essential to establish a set of criteria by which the validity of the animal model can be judged. The clinical findings which have been associated with occupational asthma are listed in table 1. Various combinations of these symptoms might be expected in individual cases, with variation dependent upon factors, such as the nature of the actual allergen, the occupational setting, the length of exposure, etc.

Animal models of occupational asthma could be expected to simulate some of these clinical observations. For example, airflow disturbance, impaired lung function, airway hyperreactivity, bronchial eosinophilia, and other physiological and pathological features could be determined in animal species. However, some of the more subjective findings could not be simulated, such as chest tightness, dyspnoea, and perhaps cough.

Parameters appropriate for the study of occupational asthma in animal species are suggested in table 2 . Whilst it is apparent that numerous factors could be measured in animals, it is also clear that in isolation, none of the items would be sufficient to reach the conclusion of occupational asthma. Rather, a selected combination of factors would appear to be more appropriate. Furthermore, selection of the particular combination of parameters should be based on the purpose of the investigation. For example, if the objective were to determine the involvement of particular cytokines in the late-phase response, appropriate parameters to establish in the model would probably include: pulmonary eosinophilia, airway hyperresponsiveness, altered airflow, etc. IgE level, cyanosis, and spasmogen release would not be helpful. Thought must be directed toward selection of the appropriate animal species and the parameters to be assessed.

\section{Caveats in use of animal models}

With all the benefits to be derived from the development and use of animal models for study of occupational asthma, there exist caveats. Attention must always be 
given to identifying differences between animal and human systems. Anatomical, physiological and biochemical differences exist between all species. Characteristics, such as airway morphometry, breathing patterns, innervation of respiratory tract tissue, and circulation to the lung, are critical to sensitization and to asthma. These factors, along with immunological components, must be considered in evaluation of an animal model.

Bronchus-associated lymphoid tissue (BALT). The occurrence and function of BALT differs among animal species. Indeed, BALT may not be present in all species [17]. BALT is organized lymphoid tissue, covered by a flattened lymphoepithelium, located within the bronchial submucosa. It is morphologically similar to gutassociated lymphoid tissue (GALT). BALT contains no goblet cells and is heavily infiltrated.

Humans, nonhuman primates and dogs have little BALT [18]. When described in humans, the tissues which had been examined were diseased, for example neoplastic lung, or inflammatory disease [18]. In humans, it has been described as "diffuse collections of subepithelial lymphoid tissue in the bronchi, with limited amounts in the small bronchi and bronchioles" [18].

Differences have been identified in the response of BALT from various species to lung immunization [18]. The relative presence or absence of BALT may influence antigen handling and processing. BALT represents a major factor to consider in selection of a particular animal model for elucidating factors related to asthma.

High versus low molecular weight allergens. Numerous animal models of pulmonary hypersensitivity have been developed and recently evaluated [19]. Considerable differences exist among models in the following aspects: the animal species utilized; the route of antigen administration; protocol for both induction and elicitation of responses; type of response measured; and criteria for conclusion of "significant" reactions.

It should be emphasized that most models of asthma, and specifically occupational asthma, were developed using high molecular weight (HMW) allergens, notably proteins, and particularly ovalbumin. Few attempts have been made to adapt these models to low molecular weight (LMW) allergens. A notable exception is the guineapig model of toluene diisocyanate (TDI) asthma [20].

\section{Animal models}

Many species have been utilized in the development of animal models of asthma, including mice, rats, guineapigs, hamsters, dogs, sheep, horses and monkeys. Each possess certain advantages and disadvantages as a model of asthma.

\section{Murine models}

A murine model for asthma presents numerous advantages when compared with use of other animal species. A comparison of species' characteristics is given in table
3. Adult mice weigh approximately $20-25$ g. Being small, they are easy to handle and relatively inexpensive. $\operatorname{IgE}$ is the major anaphylactic antibody in the mouse, making this species appropriate for investigation of the role of humoral immune factors in development of asthma. A mouse model offers the opportunity to explore mechanisms of allergic reactions because of the existence of numerous immunological reagents specific for murine cytokines, growth factors and cell surface receptors. The many inbred murine strains permit purification and transfer of materials from sensitized to naive animals for assessment of functional ability of specific factors and cells. For these reasons, it is expected that major advances in the molecular biology of sensitization will probably arise from murine studies. On the other hand, because of considerable physiological dissimilarities with primates, the ability to extrapolate the murine findings to humans will be difficult.

There are disadvantages to selection of a murine system for study of asthma. Foremost, is the recognition that the vasculature, rather than the lung, is the primary target of anaphylactic response. This disadvantage of the mouse, together with its poorly developed airway musculature, suggests that developing a physiological model of pulmonary responsiveness would be inappropriate in the mouse.

Murine models have provided fundamental information regarding certain features of asthma. Identification of particular lymphocyte subpopulations associated with asthma has been obtained using mice. Lymphocytes in the bronchoalveolar lavage of mice were investigated using flow cytometry [21]. T-helper (Th) cells predominated both over T-suppressor cells and B-cells. However, the increase noted was not proportional to the changes in the lung function of animals.

Involvement of T-lymphocytes in airway hyperreactivity was concluded from studies in mice. Trachea isolated from antigen-challenged athymic mice did not demonstrate increased response to carbachol compared with those from nonsensitized mice [22]. However, transfusion of naive mice with lymphoid cells from immunized donors restored the in vitro airway hyperreactivity. In vivo airway hyperreactivity following transfer of spleen and lymph node cells from immunized donor mice was also shown.

A murine model of airway hypersensitivity has been described [23], in which measurement of $\operatorname{IgE}$ is used as the determinant factor. The model is based on the premise that all chemical allergens have the ability to cause dermal sensitization, but only a subset of these chemicals has the ability to additionally cause airway hypersensitivity. The model assesses the potential to cause airway hypersensitivity by noting which T-helper cell subset is stimulated following exposure to the test allergen. In BALB/c mice, IL-4 exerts a dominant influence on the early, antigen-stimulated response of CD4+ T-cells [24]. The result of antigen-stimulated differentiation of Th populations to Th2 rather than Th1 cells is the production of high levels of $\mathrm{IgE}$.

The hypothesis underlying use of this methodology is that respiratory allergy is an immediate type response, whereas contact hypersensitivity is a delayed type response 
Table 3. - Advantages and disadvantages of various animal models of asthma

Advantages

\section{Mouse}

Small, inexpensive animal

Numerous inbred strains

Availability of species-specific reagents

$\mathrm{IgE}$ is major class of anaphylactic antibody

Demonstrates AHR to carbachol

Rat

Small, inexpensive animal

$\mathrm{IgE}$ is major class of anaphylactic antibody

Demonstrates EAR and LAR

Demonstrates AHR lasting for days

Responds to methacholine

Responds to cromolyn

\section{Guinea-pig}

Small, docile animal

Relatively inexpensive

Sensitization possible via inhalation rout

Lung is major shock organ

Airways respond to histamine

Demonstrates EAR and LAR

Neutrophil influx to lung following LAR

Eosinophilic inflammation during LAR

AHR following airway hypersensitivity response

Tracheal smooth muscle responds to histamine

\section{Rabbit}

Lung is anaphylactic target organ

$\mathrm{IgE}$ is major class of anaphylactic antibody

Demonstrates EAR, LAR

Dog

IgE is major class of anaphylactic antibody

Natively sensitized to Ascaris

Demonstrates AHR

\section{Basenji greyhound dog}

Persistent AHR

Very responsive to methacholine

Pony

Native sensitization to "barn" environment

Develops AHR (only during clinical disease)

Recurrent exacerbations of clinical disease

Sheep

Natively sensitive to A. suum

Demonstrates EAR and LAR

Demonstrates AHR to carbachol

\section{Nonhuman primate}

Consistent response to antigen

Natively sensitized (A. suum)

Allergic responsiveness persists for years

$\mathrm{IgE}$ is major class of anaphylactic antibody
Vasculature is anaphylactic target

Poorly developed airway smooth muscle

Responds poorly to histamine

Injection of antigen required for sensitization

Adjuvants (alum, Bordetella pertussis) required for sensitization

Responds poorly to histamine

Tracheal smooth muscle responds poorly to peptide-leukotrienes

Few inbred strains exist

Few species-specific reagents

$\mathrm{IgG}_{1}$ is the major class of anaphylactic antibody

Not sensitive to cromolyn

Neonatal immunization required for LAR

Individual variability in responsiveness to A. suum

LAR not demonstrated

AHR not associated with clinical disease

Large animal, costly

Large animal, costly

Costly species

AHR: airway hyperresponsiveness; EAR: early airway response; LAR: late airway response; IgE: immunoglobulin E.

[25]. Justification for this conclusion is based on the recognition that $\operatorname{IgE}$ antibodies have been identified to all respiratory allergens, although they may have been detected in only a minor fraction of symptomatic individuals.

The model involves topical application of a range of concentrations of test chemicals to BALB/e mice. Draining lymph nodes are subsequently isolated and evaluated for activation (proliferative response). Serum is collected for antibody and immunoglobulin analyses. It is recognized that, in this model, both respiratory and dermal sensitizers produce IgG responses. However, the two classes of allergens are said to be distinguished due to the higher production of total $\operatorname{IgE}$ by respiratory, as compared with dermal sensitizers. 
Use of the BALB/c strain is required in this model. Apparently, BALB/c mice differ from most other inbred mouse strains in the relative amounts of the two cytokines, IL-4 and interferon- $\gamma$, produced upon antigen exposure. The same stimulus applied to a $\mathrm{C} 3 \mathrm{H} / \mathrm{HeN}$ strain results in a predominant Th1 response, with heightened release of interferon- $\gamma$ rather than of IL-4. The response to the same chemical in the $\mathrm{C} 3 \mathrm{H}$ mouse would be a delayed hypersensitivity type response, whereas in the BALB/c strain, the result would be a strong humoral response. This model provides an example of the differences in immune function which may exist among strains of a species.

\section{Guinea-pig models}

The guinea-pig has been used as an animal model of pulmonary hypersensitivity for more than 90 yrs [26]. As indicated in table 3 , it has demonstrated both earlyand late-phase airway constrictive responses, hyperreactivity towards cholinergic agonists, and production of hypersensitivity antibodies, including $\operatorname{IgG}_{1}$ and $\operatorname{IgE}$ [15]. Moreover, through passive transfer of purified immunoglobulins, the guinea-pig has established the role of hypersensitivity antibody in initiation of early-onset hypersensitivity, reactions and eosinophilic airway inflammation [27]. An evaluation was made [28] of the appropriateness of guinea-pig models of sensitization for quantitative prediction of hazard to man and, therefore, of risk assessment.

The benefits and disadvantages of the use of a guineapig model are listed in table 3 . The major benefit is the lung as the target organ of a hypersensitivity response. Further, both immediate- and late-onset pulmonary responses have been readily produced in this species, thus allowing mechanistic investigation of each reaction, as well as of the relationship between the two responses. The occurrence of pulmonary inflammation consistent with asthma, and composed of both eosinophilic and neutrophilic monocytes, following a hypersensitivity response, allows investigation of the role of inflammation in various physiological responses including airway hyperreactivity. Guinea-pigs are small, docile animals and relatively inexpensive.

There are considerable disadvantages to the use of the guinea-pig as an animal model. The sparsity of inbred strains (Strains \#2 and \#13 are available) prevents meaningful investigation of the genetic influence on susceptibility to sensitization and development of asthma. In addition, few species-specific reagents exist, making it difficult to identify and isolate particular cell types, such as lymphocyte subsets. Another major disadvantage of the guinea-pig is the predominance of $\mathrm{IgG}_{1}$ rather than $\mathrm{IgE}$, as the major class of anaphylactic antibody. This presents difficulty in studies of mechanisms of humoral responses to allergens.

A guinea-pig model has been developed for assessment of allergenicity of occupational agents [15, 19, 29-31], and has received rigorous review [32]. The model assesses a chemical's ability to cause airway hypersensitivity via a "relevant" route of exposure. A diagram of the model is shown in figure 2. The model utilizes inhalation as the route of exposure to the allergen, for both the sensitization and the elicitation phases of the response. Pulmonary

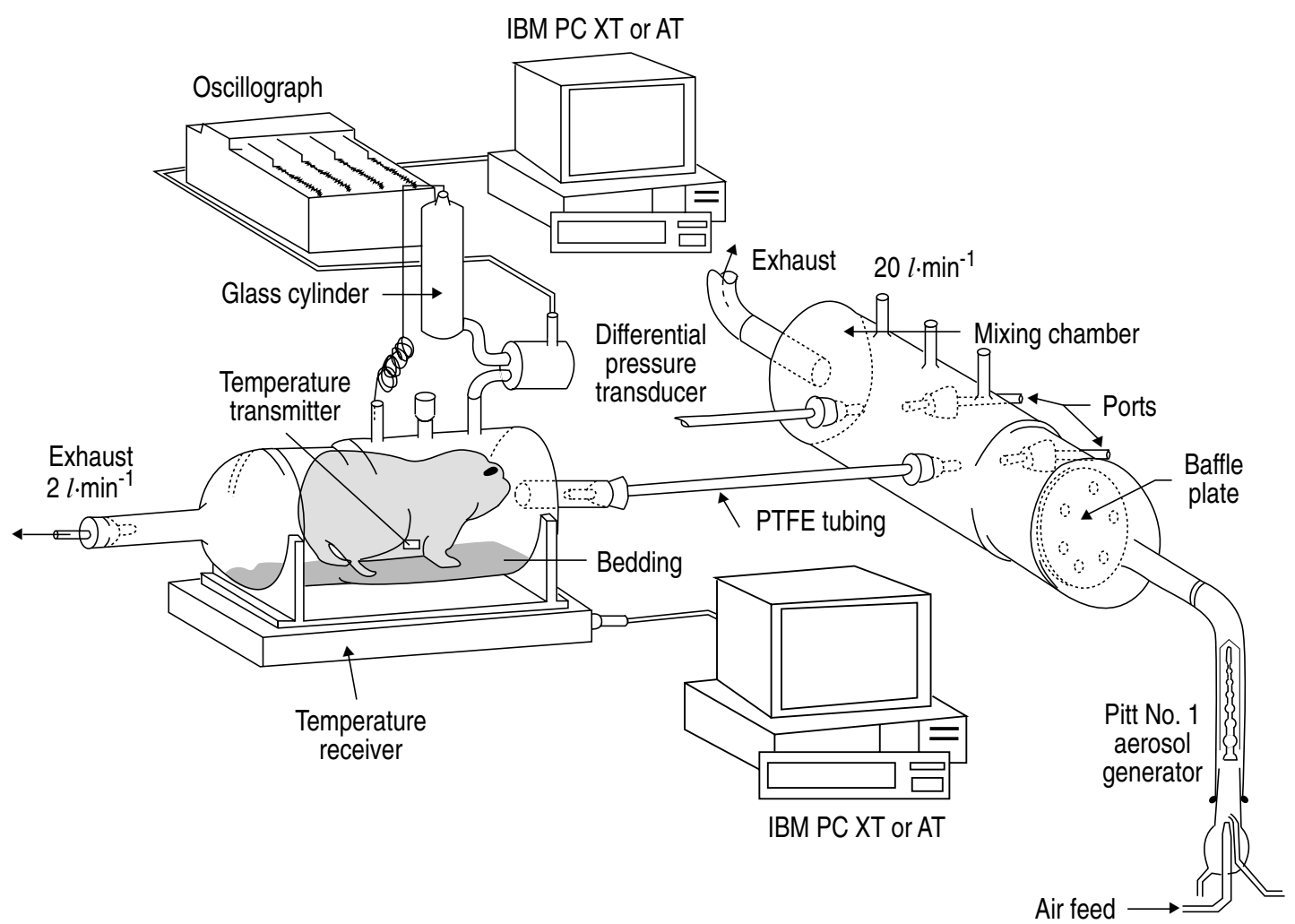

Fig. 2. - Schematic diagram of the system used for sensitization, provocation challenge, and monitoring responses of a guinea-pig to an airborne allergen. From [33], with permission. PTFE: polytetrafluoroethylene. 
function is measured using plethysmography. The latter procedure avoids the need for both anaesthesia and physical restraint of animals. It allows observation of the breathing pattern of animals, which is used to detect airway constriction, the major parameter employed to indicate sensitization. A further benefit from use of whole body plethysmography is the ability to monitor breathing patterns continuously over a period of $24 \mathrm{~h}$. Because airflow obstruction in patients may occur hours after cessation of exposure, the continuous monitoring of breathing pattern is an essential feature of the guinea-pig model to identify late-onset responses [34]. This feature has been consistently detected in populations of sensitized guineapigs [35].

For HMW protein allergens, the sensitization protocol involves inhalation of aerosols of the protein for $10 \mathrm{~min}$ a day on each of five consecutive days. Since the objective of the studies has usually been assessment of the allergenic potential of the protein, neither adjuvants nor parenteral administration of the material is used. These procedures are unnecessary in guinea-pigs and may influence the nature and severity of the resulting response.

Assessment of hypersensitivity is made three weeks following the initial day of exposure. Animals are again placed in the $2 l$ whole body plethysmographs and reexposed to the protein aerosol. During the challenge exposure and for $20 \mathrm{~h}$ thereafter, the breathing pattern of the animal is recorded. The occurrence of early- and late-phase reactions is detected by changes in breathing frequency and evidence of airway constriction. An example of the early-onset reaction is displayed in figure 3 [36]. In this example, the frequency of respiration is seen to increase within $1.5 \mathrm{~min}$ of exposure of the sensitized animal to ovalbumin. Shortly thereafter, the frequency decreases and airflow obstruction can be detected. The latter is indicated by the pattern (fig. 3) of a prolonged exhalation phase of the breath.

The severe early-onset bronchoconstrictive responses of guinea-pigs provide both advantages and disadvantages to the guinea-pig model. The advantage is the existence of a sensitive end-point for assessment of allergenic potency of agents, and for testing of efficacy of anti-spasmatic drugs. The disadvantage is the frequent lethality of the airway constrictive reactions. To avoid the latter, and allow detection of possible late-phase reactions, two procedures have been employed. Firstly, antihistamine drugs, such as mepyramine have been administered to animals approximately $30 \mathrm{~min}$ prior to the inhalation challenge with antigen [35]. This procedure lessened the airway constrictive response and did not appear to affect the increase in breathing frequency. A second method was developed [31], which utilized a "stepped dose" provocation challenge. Animals were exposed to gradually increasing concentrations of antigen for set periods of time, until reactions were observed. The method was very effective in eliciting reactions and preventing severe airway constrictive responses. It had the additional advantage that pharmacological intervention was not necessary to prevent lethality.

Demonstration of late-phase reactions gains importance in an animal model of asthma, since it bears clinical relationship to asthma. However, it is frequently difficult to identify late-phase reactions because of their characteristic gradual onset and recovery. In models of asthma associated with low molecular weight chemicals, detection of these responses is particularly important since this is the most prevalent type of response to LMW chemicals [6]. The guinea-pig model depicted in figure 2 has been able to demonstrate late-onset reactions. An example of such is provided in figure 4 . The upper pattern represents the breathing frequency of a sensitized guinea-pig prior to, and for $17.5 \mathrm{~h}$ following, inhalation provocation challenge with ovalbumin. The animal is seen to have an early-phase reaction, which occurred during the period of challenge. The respiratory rate increased from a prechallenge baseline of 75 breaths $\cdot \mathrm{min}^{-1}$ (bpm) to a maximum of $185 \mathrm{bpm}$ (a $146 \%$ increase). A late-phase reaction is also apparent, with onset at approximately $6 \mathrm{~h}$ and continuing for approximately $1.5 \mathrm{~h}$. The temporal nature of this dual reaction is similar to that noted clinically.

Occasionally, its characteristic gradual onset makes the late-phase response difficult to identify. To assist in its recognition, assessment of additional parameters is made. Since late-onset reactions are believed to represent a mild inflammatory response in the lung, the animal model incorporates monitoring of febrile reactions. Temperature-sensitive radio transmitters are implanted into the peritoneal cavity of animals prior to the start of the sensitization process. Radio signals are detected by receiver boards placed beneath each plethysmograph (fig. 2 ). As seen in figure 4 , the febrile response has been found to accompany the late-phase reaction [35]. Its

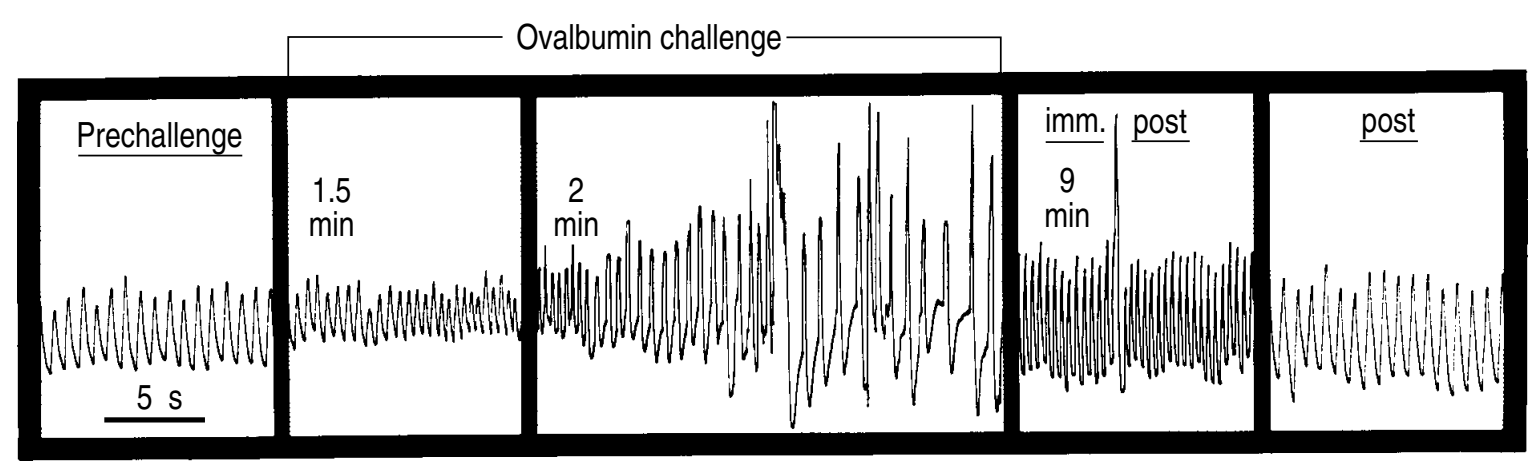

Fig. 3. - Breathing pattern of a sensitized guinea-pig an early-onset reaction to the inhaled protein allergen, ovalbumin. Within 1.5 min of inhalation, the respirations were faster, and more shallow. Maximum response was noted at 3 min and consisted of deep breathing with prolongation of the expiratory phase of the cycle. Recovery was incomplete by 9 min (immediately post-exposure). From [36], with permission. 

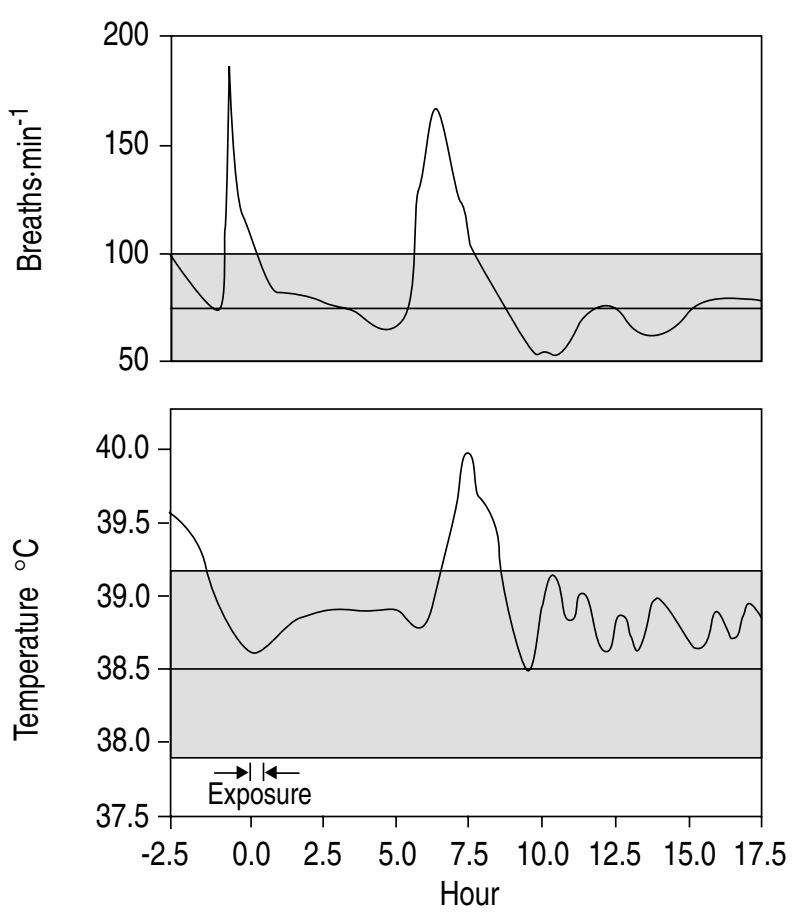

Fig. 4. - Respiratory and febrile responses of a sensitized guinea-pig upon inhalation provocation challenge with allergen. A dual respiratory response was noted as seen in the upper figure. A febrile response occurred at $7 \mathrm{~h}$ and coincided with the late-phase respiratory reaction.

occurrence can, therefore, provide confirmation of the latter response.

Airway hyperreactivity, a cardinal feature of clinical asthma, is also measured in the animal system (table 4). The degree of hyperreactivity is assessed by increased pulmonary responsiveness of animals to inhaled histamine [36].

A serological response is additionally assessed in the guinea-pig model. Blood is taken from animals prior to sensitization, and again just prior to inhalation challenge.
The serum is evaluated for specific antibody, typically using enzyme-linked immunosorbent assay (ELISA). For detection of antibody to low molecular weight (haptenic) allergens, protein conjugates of the hapten are used in ELISA. These conjugates have been prepared by reaction of the hapten with serum albumin, attempting to achieve covalent binding of 20-30 moles hapten per mole protein.

The model has been validated in two ways. The model has been established in numerous laboratories and, in each, responses of animals to a standard respiratory sensitizer, TDI, have been similar. This confirms the robustness of the model. Secondly, the model has accurately distinguished pulmonary sensitizers from nonpulmonary sensitizers. For example, inhalation exposure of animals to TDI and diphenylmethane diisocyanate (MDI) resulted in pulmonary sensitization, whereas similar exposure to formaldehyde and hydrogenated MDI, two recognized contact sensitizers, resulted in dermal sensitivity of guinea-pigs. Validation achieved with the animal model engenders confidence that future mechanistic studies will yield information applicable to human disease.

Several variations of the model have been described $[30,32]$ in attempts to either simplify the induction process (i.e. avoid the need to generate and monitor chemical atmospheres) or the elicitation phase (by monitoring EAR exclusively). Alternatives to inhalation models are desirable when there is difficulty generating stable atmospheres of reactive chemical allergens. Such is the case for certain isocyanates, such as MDI. Injection models have been used in these circumstances $[30,32]$. In these procedures, primary exposure to the chemical is by injection, administered intradermally or intraperitoneally (i.p.). Thereafter, serological evaluation may be performed to assess anaphylactic antibody as a parameter of response. Respiratory sensitivity, if assessed, utilizes a protein conjugate of the reactive chemical hapten, which is administered by intratracheal or inhalation exposure of the guinea-pig [28].

Table 4. - The guinea-pig model* of respiratory hypersensitivity

Feature

Benefit

Animals are nonsedated

Animals are nonrestrained

Inhalation route for sensitization

Inhalation route for challenge

Monitor development of EAR and LAR

Detects hyperreactive airways (AHR)

Continuous passive monitoring for mild febrile reactions

Assessment of specific and nonspecific antibody formation

Assessment of dermal sensitivity

Histological evaluation of pulmonary tissue

*: the model is described in [19, 33, 35]. AHR: airway hyperreactivity; APC: antigen-presenting cell; EAR: early-onset airway response; LAR: late-onset airway response.
Avoids effects of anaesthetics on breathing control and patterns

Allows measurement of unaltered breathing pattern for at least $24 \mathrm{~h}$

Direct assessment of chemical's ability to cause response in respiratory tract Since mechanism of LAR unknown, allows detection of both types of response, continuous monitoring of respiratory pattern allows recognition of irritant effect of chemical exposure

AHR associated with LAR and is a cardinal feature of asthma

Fever has been associated with inflammation and LAR, passive procedure does not affect measurements of breathing pattern

Determine effects on humoral and cellular elements of immune system, compare potency of chemical toward skin $v s$ respiratory system Assessment of chemical damage to lungs resulting from sensitization or challenge exposures, composition of inflammatory cells and cytokine response essential to mechanistic interpretation of response
Utilizes natural exposure route, permits access of allergen to appropriate APC

Determine effects of chemical exposure on the humoral immune system 
Mechanistic studies. The mechanism underlying the latephase reaction has been investigated in the guinea-pig. The ovalbumin-sensitized guinea-pig model was selected, since it has been shown to demonstrate both early- and late-phase asthmatic reactions, as well as airway eosinophilia, and bronchial hyperreactivity. Studies were undertaken to assess factors responsible for development of airway eosinophilia [37, 38]. Dunkin-Hartley guineapigs were sensitized by i.p. injection of ovalbumin. Three days before commencing inhalation challenge, animals were administered anti-IL-5 antibodies, using encapsulated hybridoma cells, which produced rat monoclonal antibodies to murine IL-5. One week later, guinea-pigs received a second injection of hybridoma cells. Beginning 14 days later, they were challenged daily for 1-3 min with OA aerosol, or until signs of respiratory distress. Results indicated a role for IL-5 in allergic bronchial eosinophilia. The BAL eosinophilia produced as a result of the OA inhalation challenges was greatly reduced in animals that had received the anti-IL-5 antibody. By contrast, the neutrophilia was not reduced.

The role of eosinophils in airway hyperreactivity was assessed using the guinea-pig model [39]. OA-sensitized animals developed airway hyperreactivity as a result of daily challenge with $\mathrm{OA}$ aerosol on eight consecutive days. Airway hyperreactivity was assessed as in vitro tracheal ring contractions. A role for IL-5 in airway hyperreactivity was suggested by the finding that the anti-IL-5 antibody completely inhibited the tracheal reactivity of the OA challenged animals [39].

Confirmatory evidence was obtained from experiments in which animals were treated with IL-5. Increased tracheal reactivity was noted. Hyperreactivity was apparent prior to histological visualization of eosinophilia. It was concluded that IL-5 had considerable significance in the hyperreactivity, but the role of eosinophils in the hyperreactivity was uncertain. Neutrophils did not appear to be of importance in hyperreactivity, since the neutrophil increase was not affected by anti-IL- 5 treatment.

The strong bronchoconstrictive response noted in guineapigs makes it an excellent model with which to study the late-phase response and bronchial hyperreactivity. The early- and late-phase reactions, together with the eosinophilia (increased proportion of hypodense eosinophils, as in human asthmatics) and hyperreactive airways, makes it a relevant model.

\section{Rats}

The rat represents a third small rodent species, which has received considerable attention for use in development of a model of asthma. Benefits from use of a rat model are summarized in table 3 . The production of $\operatorname{IgE}$ as the major anaphylactic antibody enables studies on the site of synthesis of allergen-specific antibody, as well as on the role of antibody in the physiological and cytological responses to allergen. Both the early- and late-phase reactions have been produced in strains of rats; however, sensitization typically requires injection of the allergen [40, 41], rather than its administration via the inhalation route as is used with guinea-pigs. In addition, allergic sensitization requires use of adjuvants, specifically, alum and Bordetella pertussis [40-42]. This can be a considerable disadvantage in the study of allergic potencies of chemicals, and for study of mechanisms of sensitization. Another benefit from use of a rat model is the ability to produce long lasting (several days) airway hyperreactivity. The species responds well to methacholine, but poorly to histamine and peptide-leukotrienes.

A model of allergic bronchoconstriction has been described using the Donryu rat [40]. The model employs i.p. injection with antigen (ovalbumin), together with killed $B$. pertussis. Two to three weeks later, animals are anaesthetized and challenged by injection of antigen into the jugular vein. Response parameters include airway resistance and IgE titre. Wistar, Lewis, DA, and Donryu rats were tested. The Donryu strain demonstrated increased airway resistance of 70-90\%, compared with 20-40\% for the other strains. The airway resistance shown by the Donryu strain was "biphasic", with both responses occurring within $6 \mathrm{~min}$. The first phase began approximately $1 \mathrm{~min}$ following challenge, and the second $1.5-6 \mathrm{~min}$ after challenge. There was minimum recovery between responses.

The severity of the bronchoconstriction was dependent upon the antigen dose, and correlated with the titre of specific IgE antibody in the serum [40]. Bronchoconstriction was rarely provoked if the IgE titre, assessed using passive cutaneous anaphylaxis assay, was lower than 16. The airway resistance response was inhibited by aminophylline and cromolyn sodium and was thought to be caused by serotonin release from mast cells or basophils.

Early and late airway responses (EAR and LAR) have also been observed in the Brown-Norway rat [41, 42]. The early response occurred within $5 \mathrm{~min}$ of challenge exposure; the late-onset response between 120-600 min after challenge. Compared with the Donryu strain, onset of the late response in this model more closely parallels the human response. In the study by EIDELMAN et al. [42], 7 of the 10 animals which gave the early response also had a late response. There was no difference in the magnitude of the EAR between the group with LAR and the group without LAR. The development of LAR was not associated with changes in airway responsiveness to methacholine (assessed one week after challenge). In this study, the one week delay was necessary to allow adequate recovery from prolonged anaesthesia and intubation. However, it is recognized that the responsiveness may have been missed because of the delay.

Bellofiore and MARTIN [41] succeeded in sensitizing $70 \%$ of rats as assessed by increase in pulmonary resistance (RL) of 70-450\% (averaging 264\%). Sensitization did not result in increased airway responsiveness as assessed by inhalation of doubling doses of methacholine followed by measurement of RL. However, after about three antigen challenges (repeated at 5 day intervals) rats were more responsive to methacholine, and remained so for approximately 7 days. Nonresponders to antigen did not show airway hyperreactivity. Moreover, the increased cholinergic responsiveness occurred only in animals that developed EAR. 
A frequent occurrence in experimental models of asthma is a tendency toward desensitization of animals with continued antigen challenge. This has been noted in rats and in guinea-pigs. The mechanism of the desensitization is unknown. However, in guinea-pigs, there was a decreased ability of antigen to release histamine from lung homogenates of animals that had undergone repeated challenges [43].

The role of cytokines in airway inflammation and hyperreactivity was studied in a rat model. Inhalation of TNF caused neutrophilic infiltration into the BAL and hyperresponsiveness to serotonin (5-HT) [44].

Being a small rodent, the rat offers economical advantages. In addition, the Brown-Norway rat produces high levels of specific $\operatorname{IgE}$ antibody during sensitization, thus allowing assessment of the role of $\operatorname{IgE}$ in sensitization, and asthmatic processes. If sensitization could be achieved without adjuvants, and the physiological response to allergen challenge could be elicited without injections and anaesthesia, rats would provide distinct advantages as a model appropriate for study of mechanisms of, and genetic influence on, susceptibility to asthma.

\section{Rabbits}

The rabbit provides an animal model which resembles humans in that the lung is the target organ for anaphylactic response. This species can demonstrate both early- and late-onset reactions. The latter's association with inflammation is thought to be of greatest importance in the development of asthma. The rabbit is a valuable model, since it also produces IgE as the primary anaphylactic antibody (table 3).

In the rabbit model, neonatal rabbits, immunized within $24 \mathrm{~h}$ of birth with an extract of Alternaria tenius, demonstrated IgE antibodies to the mould [45]. The animals respond to inhalation challenge with a biphasic lung response characterized by increased $\mathrm{RL}$ and decreased dynamic compliance (Cdyn). The early-phase changes in $\mathrm{RL}_{\mathrm{L}}$ and Cdyn usually begin within $15 \mathrm{~min}$ of the challenge (5 min duration). The second reaction typically begins by $100 \mathrm{~min}$, and persists through the $6 \mathrm{~h}$ monitoring period.

In this model, both the early and late phase reactions have been associated with IgE. Transfer of serum containing antibody solely of the IgE class into naive animals reproduced both responses upon aerosol challenge of the passively sensitized animals. Infusion of specific $\mathrm{IgG}$ antibodies reduced or abrogated the response to antigen [46]. These studies indicate the ability to produce a late-phase response in the absence of cellular immune components.

\section{Dogs}

The dog represents a model in which sensitization occurs as a result of natural exposure. An IgE-mediated model of asthma has been described which results from natural infection of dogs with Ascaris [47]. Animals demonstrate early-phase responses and airway hyperreactivity.

Another dog model involves immunization of dogs within one month of birth with ragweed and mixed grass pollen extracts [48]. Bimonthly injections, thereafter, resulted in maintenance of high IgE levels. Upon aerosol challenge, animals reacted within $5 \mathrm{~min}$ and displayed increased pulmonary resistance. A late-phase response was not described. Airway hyperreactivity was increased at $6 \mathrm{~h}$ and persisted for up to 4 months. Regarding the mechanisms of the hyperreactivity, inflammatory cells were noted in BAL (performed at $6 \mathrm{~h}$ ) of dogs showing airways hyperreactivity (AHR). In dogs which responded to antigen, but did not show AHR, no significant changes in these cell types were noted in the BAL. In this model, it is concluded that airway response to antigen is influenced by the amount of bronchoconstrictive agent released, by baseline nonspecific airway responsiveness, and by vagal reflex mechanisms [48].

A Basenji greyhound model of asthma presents valuable and unusual features (table 3). The model utilizes dogs natively sensitive to Ascaris suum. For pulmonary evaluation, dogs are anaesthetized and supported by a sling. Animals show increased pulmonary resistance and decreased dynamic compliance following challenge with aerosolized antigen [49]. Ascaris-induced bronchoconstriction resulted in a 9-20 fold increase in RL.

The Basenji greyhound dog has persistent marked bronchial hyperreactivity and, thereby, mimics human asthma. The animals are 10-30 times more sensitive to methacholine than mongrel dogs. A correlation was not apparent between responsiveness to Ascaris challenge and methacholine reactivity. A similar lack of association was noted in Ascaris-sensitive Rhesus monkeys.

The model has been used to probe the mechanisms underlying airway responsiveness to antigen. In an investigation of the relationship between antigen-specific serum $\mathrm{IgE}$, blood leucocyte histamine release and pulmonary responses to Ascaris challenge, pulmonary responses correlated more closely with the antigen dose required for in vitro release of histamine from leucocytes, than with the circulating $\operatorname{IgE}$ titre ( $\mathrm{r}=0.94$ versus $\mathrm{r}=0.68$ ) [50]. The findings imply that response to antigen challenge is more dependent upon releasability of mediators from pulmonary cells than upon the level of IgE.

\section{Horses and ponies}

Horses and ponies naturally develop a respiratory disorder characterized by acute airway obstruction followed by periods of disease remission [51]. Clinical manifestations, accompanied by changes in lung function, occur when animals are exposed to hay or barn dust. Clinically affected horses are typically hyperreactive to inhaled histamine [52]. An allergic aetiology has been assumed, due to the presence of serum antibodies and response to aerosol antigen provocation challenge. Affected horses are hypoxaemic, have reduced Cdyn and increased $\mathrm{R}_{\mathrm{L}}$, bronchiolitis, goblet cell metaplasia, airway smooth muscle hypertrophy, and excess mucus and inflammatory cells 
in the airways. Lesions in larger airways are not typical. Tachypnoea and reduced tidal volume occur during acute heaves.

This naturally occurring disorder resembles human asthma in its spontaneous occurrence. With regard to nonspecific airway hyperreactivity, in the model, airway hyperreactivity disappears during clinical regression. With occupational asthma, the concordance is not absolute [53].

\section{Sheep}

The sheep has provided a model in which to study both the early- and late-phase responses to allergen. The sheep develop a natural cutaneous and respiratory reactivity to A. suum. Allergic sheep can be divided into early responders, and dual responders [54]. Dual responders were shown to develop allergen-induced AHR to carbachol at $24 \mathrm{~h}$, whereas sheep with isolated early responses did not [54]. AHR had returned to normal by 3 weeks.

Experimentally, sheep are unsedated and restrained in a prone position. Animals are intubated with an endotracheal tube and aerosol delivered into the tube for challenge. Nonimmunological challenges of allergic sheep with compound 48/80, a mast cell degranulator, induced an immediate and late-phase reaction. Such evidence suggests involvement of mast cell mediators in the pulmonary late-phase response.

\section{Nonhuman primates}

Possessing natural sensitivity to Ascaris antigen, the Rhesus monkey provides a model of IgE-mediated acute allergic airway response [55]. The animals demonstrate both skin and respiratory reactivity to the antigen, and develop hyperreactive airways to histamine and carbacholine [56].

Populations of monkeys have been identified which are analogous to human IgE-mediated allergic populations. These include: 1) monkeys that have persistent and consistent IgE-mediated cutaneous and asthmatic responses to Ascaris antigen; 2) monkeys which have cutaneous and airway reactivity to Ascaris antigen but in which the airway reactivity subsides and disappears (analogous to a spontaneous remission of human asthma); and 3) animals with cutaneous reactivity but no asthma [57]. These groupings are analogous to: normal humans; humans with IgE antibody and no asthma; and humans with IgEtriggered asthma. Over a period of $24 \mathrm{yrs}$, animals were consistent in the type of response they displayed.

In this model, antigen challenge is given by aerosol. The protocol is to give either a maximum concentration or increasing concentrations, each for $10 \mathrm{~min}$ until a reaction is observed. For measurement of airway responses, animals are anaesthetized and fitted with an endotracheal tube. The parameters measured include: respiratory frequency $(f)$, pulmonary resistance $\left(\mathrm{R}_{\mathrm{L}}\right)$, peak expiratory flow rate (PEFR), tidal volume $\left(\mathrm{V}_{\mathrm{T}}\right)$, and dynamic compliance (Cdyn). Results are expressed as percentage change from baseline, with a minimum response defined as $f+20 \%$; RL $+25 \%$; Cdyn -20\%; PEFR -25\%; $\mathrm{V}_{\mathrm{T}}$ $-15 \%$. A minimum positive response is one in which four of the five parameters is positive. With this model, it is the number of abnormal responses which is significant, rather than the degree of response of any single parameter.

Naturally sensitized, conscious squirrel monkeys have demonstrated immediate and late-phase responses upon aerosol challenge with Ascaris antigen [58]. Aerosol challenge was given through a face mask for a $6 \mathrm{~min}$ period. RL and Cdyn were measured for each $5 \mathrm{~min}$ interval prior to challenge and for $1 \mathrm{~h}$ following challenge, and every $15 \mathrm{~min}$ until $11 \mathrm{~h}$. Responses involved central and peripheral airways. Late reactions were defined by meeting the following three criteria: a reproducible second increase in RL and decrease in Cdyn in 3-4 consecutive experiments; partial recovery of at least $50 \%$ from the early reaction; and late-phase changes in Cdyn and RL having similar or greater magnitude than the early-phase response. Data suggested that the most consistent indicator of the late response in the squirrel monkeys is Cdyn.

Results of challenge with Ascaris antigen indicated that pulmonary changes occurred in the absence of any significant variations in $f$ or $\mathrm{VT}_{\mathrm{T}}$ [58]. Late responses occurred in four of nine animals which demonstrated early reactions. The four could not be distinguished from the other five by: baseline airways calibre, severity of early response to antigen, or cutaneous reactivity to Ascaris. The time course of the early- and late-phase responses was similar to the human dual reaction. Increasing the amount of antigen inhaled resulted in late-phase responses following early reactions. In no case was an isolated late-phase reaction observed.

Some of the animals demonstrated persistent asthma. Others demonstrated a progressive loss of airway reactivity over a period of years. Usually, in these animals, the cutaneous reactivity persisted with no decrease in endpoint titres.

The model has many similarities to human asthma, in that some populations have persistent allergen-triggered asthma, others have spontaneous subsidence of asthma but remain skin test positive, and others have positive skin tests with no asthma [58]. Attempts to develop a sensitive colony by deliberate parasitic infection of primates using A. suum ova, resulted in cutaneous and respiratory reactivity, however the latter typically lasted for less than $1 \mathrm{yr}$.

\section{In vitro models}

In addition to models which use whole animals, in vitro systems often provide valuable mechanistic information. Increased numbers of antigen (Ia)-positive monocytes and dendritic cells were noted in the BAL fluid from rats during a nonspecific inflammatory reaction [59]. A study was conducted to determine whether dendritic cells or macrophages were responsible for antigen presentation in vivo. Splenic macrophage were pulsed 
with antigen in vitro and, subsequently, instilled intratracheally into rats. The ability to sensitize T-cells in the draining lymph nodes of the lung was assessed. Alveolar macrophages (AMs) were obtained from the bronchiolar lavage of ACI/Mal rats. Dendritic cells (DCs) were obtained from a cell suspension of the spleen. Each cell type was cultured overnight with antigen (a copolymer of glutamine-tyrosine, which had been shown to be antigenic in this rat strain in an Ia-dependent manner). The cell populations were subsequently injected into the hind foot pads of recipient rats and injected intratracheally. Four or five days later, the popliteal or paratracheal lymph nodes, and cervical lymph nodes (draining the upper respiratory tract) were removed and cultured with antigen and examined for incorporation of tritiated thymidine. Antigen-pulsed DCs or AMs resulted in antigen-specific responses of paratracheal lymph nodes. Cervical lymph nodes gave no responses. Free antigen was ineffective. Dose-response studies demonstrated that dendritic cells were approximately 10 times more effective than AMs in inducing priming of lymph nodes.

Other studies have shown that AMs suppress antigenstimulated lymphoproliferative responses in vitro. Depletion of AMs in vivo resulted in enhanced pulmonary immune responses [60]. Dendritic cells are present in the lung, and increased numbers of Ia positive DCs and monocytes occur in the BAL during an inflammatory response [59]. Clearly, the identity of effective antigen presenting cells following a natural exposure (inhalation) to antigen needs further study.

\section{Conclusions}

The incidence of asthma has increased substantially in recent years. Cases with occupational association have also increased. The reason(s) for the rise are uncertain. It is possible that the increased dependence upon manmade chemicals, estimated to be between 1,000-2,000 new chemicals introduced into commerce each year, may be a decisive factor. Some of the chemicals are allergenic. The new materials, together with new processes utilized in their formation and application, may be factors responsible for heightened incidence of occupational asthma.

The progression from episodic occurrences of allergic sensitization to asthma has received study, but remains unclear. There is an urgent need to gain increased understanding, on a molecular, cellular and physiological basis, of the progression of the disease. Information is needed, ranging from factors contributing to the initial process of sensitization to those important for development of chronic disease and impairment of basal lung function. Further research, to probe the cellular and molecular mechanism(s) governing induction and regulation of airway hypersensitivity, will rely on prudent use of animal models.

There are advantages and disadvantages with use of each of the animal models discussed in this review. The guinea-pig model of airway hypersensitivity has been utilized for more than 90 yrs [26] to gain understand- ing of the physiological and immunological processes involved in airway hypersensitivity. The model successfully elucidated the relationship between the concentration of allergen inhaled, the titre of hypersensitivity antibody produced [15], and the occurrence of early-onset respiratory reactions [15].

The usefulness of guinea-pigs for investigation of molecular and cellular aspects of hypersensitivity appears limited. Guinea-pigs will be less effective for study of the roles of particular cytokines in development of earlyand late-phase responses, since immunological reagents to identify guinea-pig cytokines are unavailable. Similarly, identification of genes involved in regulation of the allergic response will be difficult because of the limited number of inbred guinea-pig strains. On the other hand, the availability of numerous inbred strains of mice, and immunological reagents specific to murine cytokines, suggests murine models will be favoured for the latter investigations.

Selection of the appropriate animal model for an investigation should depend upon the objective of the study. Undoubtedly, in vitro methodologies will receive greater attention in the future. This conclusion is based upon both the advances which have been made in cell purification and culture techniques and the heightened desire of researchers and government agencies to reduce the dependence on animal use in medical research, and especially in testing.

Epidemiological studies have provided data regarding characteristics of populations demonstrating hypersensitivity to high versus low molecular weight allergens [61-63]. Atopy appears to contribute to increased susceptibility to sensitization by HMW, but not LMW allergens [8, 61-63]. Other differences have been recognized in sensitization to these two classes of agents. Early-onset responses are the most frequent reactions to HMW allergens, whereas late-onset responses are more characteristic of LMW chemical agents. Such findings suggest the mechanism of sensitization may differ between the two classes of allergens. Successful mechanistic investigations of each response will depend upon prudent selection of animal models that consistently demonstrate those symptoms which are characteristics of the desired responses.

A remaining area of uncertainty in occupational asthma is the influence of environmental factors both on development of sensitivity, and on progression from episodic occurrences to chronic disease. For development of childhood asthma, much evidence has been presented for a role of upper respiratory tract viruses in initiation of the disease. Evidence also exists for contribution of air pollutants, such as ozone, nitrogen oxides and acid aerosols, to pulmonary inflammation and altered response to airborne allergens. Judicious use of animal models, together with appropriate in vitro systems, can advance understanding of environmental influences on development of occupational asthma.

Well-designed experiments utilizing animal models can provide physiological, as well as mechanistic information critical to gaining increased understanding of the disease. However, in using animal models, one must 
always keep in mind that the animal system is only a surrogate. Results from animal systems must be regularly compared with those from clinical evaluations. Investigators must be receptive to questioning differences between the human and the animal systems. Examples abound in toxicology, where adverse effects to a particular chemical were noted in certain species, and in particular organs of the species, but the same effect was never observed in humans [64]. Species differences in BALT, discussed in this review, may contribute significantly to differences between humans and certain animal species with regard to pulmonary responses. Continuous validation in humans of experimental findings is essential to avoid misinterpretations.

It is certain that continued advances in molecular immunology will result in further understanding of the aetiology of early- and late-onset respiratory hypersensitivity reactions. Development of effective in vitro screening methodologies and preventive programmes are envisaged. Until then, prudent employment of animal models will continue to guide us in recognition, treatment, and prevention of occupationally-based asthma.

Acknowledgements: The excellent assistance or G. Curtis in preparation of the manuscript is gratefully acknowledged.

\section{References}

1. Hargreave FEG, Ryan NC, Thomson PM, O'Byrne PM, Latimer K, Juniper EF. Bronchial responsiveness to histamine or methacholine in asthma: measurement and clinical significance. J Allergy Clin Immunol 1980; 68: 347-355.

2. Burrows B, Martinez FD, Halonen M, Barbee RA, Cline MG. Association of asthma with serum IgE levels and skin-test reactivity to allergens. N Engl J Med 1989; 320: 271-277.

3. Sears MR, Burrows B, Flannery EM, Herbison GP, Hewitt CJ, Holdaway MD. Relationship between airway hyperresponsiveness and serum $\operatorname{IgE}$ in children with asthma and in apparently normal children. N Engl J Med 1991; 325: 1067-1071.

4. Smith M Jr. Epidemiology and natural history of asthma, allergic rhinitis, and atopic dermatitis (eczema). In: Middleton E Jr, Reed CE, Ellis EF, Adkinson NF Jr, Yunginger JW, eds. Allergy: Principles and Practice. 3rd edn. Vol. 2. St. Louis, CV Mosby, 1988; pp. 891929.

5. Holgate $\mathrm{S}$. Mediator and cytokine mechanisms in asthma. Thorax 1993; 48: 103-109.

6. Young RP, Sharp PA, Lynch JR, et al. Confirmation of genetic linkage between atopic IgE responses and chromosome 11q13. J Med Genet 1992; 29: 236238.

7. Holt PGH. Environmental pollutants as co-factors in IgE production. Curr Opin Immunol 1989; 1: 643-646.

8. Chan-Yeung M. A clinician's approach to determine the diagnosis, prognosis, and therapy of occupational asthma. Med Clin North Am 1990; 74: 811-822.

9. Linssen MJ. Animal models for testing anti-inflammatory drugs for treatment of bronchial hyperreactivity in asthma. Pharm Weekbl (Sci) 1991; 13: 225-237.
10. Dunnill MS. The pathology of asthma, with special reference to changes in the bronchial mucosa. J Clin Pathol 1960; 13: 27-33.

11. Di Stefano A, Saetta M, Maestrelli P, et al. Mast cells in the airway mucosa and rapid development of occupational asthma induced by toluene diisocyanate. Am Rev Respir Dis 1993; 147: 1005-1009.

12. Robinson DS, Hamid Q, Ying S, et al. Predominant Th2-like bronchoalveolar T-lymphocytes population in atopic asthma. N Engl J Med 1992; 326: 298-304.

13. Iwamoto I, Nakajima H, Endo H, Yoshida S. Interferon $\gamma$ regulates antigen-induced eosinophil recruitment into mouse airways by inhibiting the infiltration of CD4+ Tcells. J Exp Med 1993; 177: 573-576.

14. Karol MH, Thorne PS, Hillebrand JA. The immune response as a biological indicator of exposure. In: Foa V, Emmett EA, Maroni M, Colombi A, eds. Occupational \& Environmental Chemical Hazards. Chichester, Ellis Horwood Ltd, 1987; pp. 86-90.

15. Karol MH. Concentration-dependent immunologic response to toluene diisocyanate (TDI) following inhalation exposure. Toxicol Appl Physiol 1983; 68: 229241.

16. Lebowitz MD, Knudson RJ, Burrows B. Family aggregation of pulmonary function measurements. Am Rev Respir Dis 1984; 129: 8-11.

17. Pabst R, Gehrke I. Is the bronchus-associated lymphoid tissue (BALT) an integral structure of the lung in normal mammals, including humans? Am J Respir Cell Mol Biol 1990; 3: 131-135.

18. Haley PJ. Immunological responses within the lung after inhalation of airborne chemicals. In: Gardner DE, Crapo JD, McClellan RO, eds. Toxicology of the Lung. 2nd edn. NY, Raven Press, 1993; pp. 389-416.

19. Karol MH. Assays to evaluate pulmonary hypersensitivity. In: Burleson G, Dean J, Munson A, eds. Modern Methods in Immunotoxicology. NY, Wiley-Liss Publishers, (in press).

20. Karol MH. The development of an animal model for TDI asthma. Bull Eur Physiopathol Respir 1988; 23: 571-576.

21. Curtis JL, Kaltreider HB. Characterization of bronchoalveolar lymphocytes during a specific antibody-forming cell response in the lungs of mice. Am Rev Respir Dis 1989; 139: 393-400.

22. Garssen J, Nijkamp FP, Van Der Vliet H, Van Loveren H. T-cell mediated induction of airway hyperreactivity in mice. Am Rev Respir Dis 1991; 144: 931-938.

23. Kimber I, Dearman RJ. The mechanisms and evaluation of chemically induced allergy. Toxicol Lett 1992; 64/65: 79-84.

24. Chatelain R, Varkila K, Coffman RL. IL-4 induces a Th2 response in Leishmania major-infected mice. $J$ Immunol 1992; 148: 1182-1187.

25. Dearman RJ, Kimber I. Differential stimulation of immune function by respiratory and contact allergens. Immunol 1991; 72: 563-570.

26. Ratner B, Jackson HC, Gruehl HL. Respiratory anaphylaxis. Sensitization, shock, bronchial asthma and death induced in the guinea-pig by the nasal inhalation of dry horse dander. Am J Dis Child 1927; 34: $23-$ 47.

27. Griffiths-Johnson D, Jin R, Karol MH. The role of purified $\mathrm{IgG}_{1}$ in pulmonary hypersensitivity responses of the guinea-pig. J Toxicol Environ Health 1993; 40: 117-127.

28. Sarlo K, Clark ED. A tier approach for evaluating the 
respiratory allergenicity of low molecular weight chemicals. Fund Appl Toxicol 1992; 18: 107-114.

29. Karol MH, Ioset HH, Riley EJ, Alarie YC. Haptenspecific hypersensitivity in guinea-pigs. Am Ind Hyg Assoc J 1978; 39: 546-556.

30. Botham PA, Rattray NJ, Woodcock DR, Walsh ST, Hext PM. The induction of respiratory allergy in guineapigs following intradermal injection of trimellitic anhydride: a comparison with the response to 2,4 dinitrochlorobenzene. Toxicol Lett 1989; 47: 25-39.

31. Hayes JP, Lotvall JO, Baraniuk J, et al. Bronchoconstriction and airway microvascular leakage in guinea-pigs sensitized with trimellitic anhydride. Am Rev Respir Dis 1992; 146: 1306-1310.

32. Sarlo K, Karol MH. Guinea-pig predictive tests for respiratory allergy. In: Dean J, Luster M, Munson A, Kimber I, eds. Immunotoxicology and Immunopharmacology. 2nd ed. NY, Raven Press, (in press).

33. Karol MH, Hillebrand JA, Thorne PS. Characteristics of weekly pulmonary responses elicited in the guineapig by inhalation of ovalbumin aerosols. Toxicol Appl Pharmacol 1989; 100: 234-246.

34. Karol MH, Stadler J, Magreni C. Immunotoxicologic evaluation of the respiratory system: animal models for immediate- and delayed-onset pulmonary hypersensitivity. Fund Appl Toxicol 1985; 5: 459-472.

35. Thorne PS, Karol MH. Association of fever with lateonset pulmonary hypersensitivity responses in the guineapig. Toxicol Appl Pharmacol 1989; 100: 247-258.

36. Griffiths-Johnson D, Karol M. Validation of a noninvasive technique to assess development of airway hyperreactivity in an animal model of immunologic pulmonary hypersensitivity. Toxicol 1991; 65: 283-294.

37. Chand N, Harrison JE, Rooney S, et al. Anti-IL-5 monoclonal antibody inhibits allergic late-phase bronchial eosinophilia in guinea-pigs: a therapeutic approach. Eur J Pharmacol 1992; 211: 121-123.

38. Gulbenkian AR, Egan RQ, Fernandez X, et al. Interleukin-5 modulates eosinophilia accumulation in allergic guinea-pig lung. Am Rev Respir Dis 1992; 146: 263-265.

39. Van Oosterhout AJM, Ladenius ARC, Savelkoul HFJ, Van Ark I, Delman KC, Nijkamp FP. Effect of antiIL-5 and IL-5 on airway hyperreactivity and eosinophils in guinea-pigs. Am Rev Respir Dis 1993; 147: 548-552.

40. Watanabe A, Hayashi H. allergen-induced biphasic bronchoconstriction in rats. Int Arch Allergy Appl Immunol 1990; 93: 26-34.

41. Bellofiore S, Martin JG. Antigen challenge of sensitized rats increases airway responsiveness to methacholine. $J$ Appl Physiol 1988; 65: 1642-1646.

42. Eidelman DH, Bellofiore S, Martin JG. Late airway responses to antigen challenge in sensitized inbred rats. Am Rev Respir Dis 1988; 137: 1033-1037.

43. Andrew KK, Schellenberg RR, Hogg JC, Hanna CJ, Paré PD. Physiological and immunological effects of chronic antigen exposure in immunized guinea-pigs. Int Arch Allergy Appl Immunol 1984; 75: 208-213.

44. Kips JC, Tavernier J, Pauwels RA. Tumor necrosis factor causes bronchial hyperresponsiveness in rats. Am Rev Respir Dis 1992; 145: 332-336.

45. Shampain MP, Behrens BL, Larsen GL, Henson PM. An animal model of late pulmonary responses to Alternaria challenge. Am Rev Respir Dis 1982; 126: 493-498.

46. Metzger WJ. Late-phase asthma in an allergic rabbit model. In: Dorsch W, ed. Late-Phase Allergic Reactions. Boca Ration, Florida, CRC Press, 1990; pp. 347-362.
47. Booth B, Patterson R, Talbot C. Immediate-type hypersensitivity in dogs; cutaneous, anaphylactic and respiratory responses to Ascaris. J Lab Clin Med 1970; 76: 181-189.

48. Chung KF, Becker AB, Lazarus SC, Frick OL, Nadel JA, Gold WM. Antigen-induced airway hyperresponsiveness and pulmonary inflammation in allergic dogs. $J$ Appl Physiol 1985; 58: 1347-1353.

49. Hirshman CA, Malley A, Downes H. Basenji greyhound dog model of asthma: reactivity to Ascaris suum, citric acid and methacholine. J Appl Physiol: Respirat Environ Exercise Physiol 1980; 49: 953-957.

50. Peters JE, Hirshman CA, Malley A. The Basenji greyhound dog model of asthma: leukocyte histamine release, serum $\mathrm{IgE}$, and airway response to inhaled antigen. $J$ Immunol 1982; 129: 1245-1249.

51. Lowell FC. Observations on heaves. An asthma-like syndrome in the horse. J Allergy 1964; 35: 322-330.

52. Derksen FJ, Robinson NE, Armstrong PJ, Stick JA, Slocombe RF. Airway reactivity in ponies with recurrent airway obstruction (heaves). J Appl Physiol 1985; 58: 598-604.

53. Cockcroft DW, Hargreave FE. Airway hyperresponsiveness. Relevance of random population data to clinical usefulness. Am Rev Respir Dis 1990; 142: 497-500.

54. Lanes S, Stevenson JS, Codias E, et al. Indomethacin and FPL-57231 inhibit antigen-induced airway hyperresponsiveness in sheep. J Appl Physiol 1986; 61: 864-872.

55. Weiszer I, Patterson R, Pruzansky JJ. Ascaris hypersensitivity in the Rhesus monkey. I. A model for the study of immediate type hypersensitivity in the primate. $J$ Allergy 1968; 41: 14-22.

56. Patterson R, Harris KE. Rhesus monkey airway responses to substance P. Int Arch Allergy Appl Immunol 1990; 91: 374-379.

57. Patterson R, Harris KE. IgE-mediated rhesus monkey asthma: natural history and individual variation. Int Arch Allergy Immunol 1992; 97: 154-159.

58. Hamel R, McFarlane CS, Ford-Hutchinson AW. Late pulmonary responses induced by Ascaris allergen in conscious squirrel monkeys. J Appl Physiol 1986; 61: 2081-2087.

59. Havenith CEG, Breedijk AJ, Betjes MGH, Calame M, Beelen RHJ, Hoefsmit ECM. T-cell priming in situ by intratracheally instilled antigen-pulsed dendritic cells. Am J Respir Cell Mol Biol 1993; 8: 319-324.

60. Thepen T, Van Rooijen N, Kraal G. Alveolar macrophage elimination in vivo is associated with an increase in pulmonary immune response in mice. J Exp Med 1989; 170: 499-509.

61. Juniper CP, How MJ, Goodwin BFJ, Kinshott AK. Bacillus subtilis enzymes: a 7 year clinical, epidemiological and immunological study of an industrial allergen. $J$ Soc Occup Med 1977; 27: 3-12.

62. Flood DFS, Blofeld RE, Bruce CF, Hewitt JI, Juniper $\mathrm{CP}$, Roberts DM. Lung function, atopy, specific hypersensitivity, and smoking of workers in the enzyme detergent industry over 11 years. $\mathrm{Br} J$ Ind Med 1985; 42: 43-50.

63. Karol MH, Tollerud DJ, Campbell TP, et al. The predictive value of airways hyperresponsiveness and circulating IgE for identifying types of responses to toluene diisocyanate inhalation challenge. Am J Respir Crit Care Med 1994; 149: (In press).

64. Hewitt WR, Goldstein RS, Hook JB. Toxic responses of the kidney. In: Amdur MO, Doull J, Klaassen CD, eds. Casarett and Doull's Toxicology: The Basic Science of Poisons. 4th edn. New York, Pergamon Press, 1991; pp. 354-382. 